\title{
Artifact Reduction of Different Metallic Implants in Flat Detector C-Arm CT
}

\author{
S.-C. Hung, C.-C. Wu, C.-J. Lin, W.-Y. Guo, C.-B. Luo, F.-C. Chang, and C.-Y. Chang
}

\begin{abstract}
BACKGROUND AND PURPOSE: Flat detector CT has been increasingly used as a follow-up examination after endovascular intervention. Metal artifact reduction has been successfully demonstrated in coil mass cases, but only in a small series. We attempted to objectively and subjectively evaluate the feasibility of metal artifact reduction with various metallic objects and coil lengths.
\end{abstract}

MATERIALS AND METHODS: We retrospectively reprocessed the flat detector CT data of 28 patients (15 men, 13 women; mean age, 55.6 years) after they underwent endovascular treatment (20 coiling \pm stent placement, 6 liquid embolizers) or shunt drainage $(n=2)$ between January 2009 and November 2011 by using a metal artifact reduction correction algorithm. We measured CT value ranges and noise by using region-of-interest methods, and 2 experienced neuroradiologists rated the degrees of improved imaging quality and artifact reduction by comparing uncorrected and corrected images.

RESULTS: After we applied the metal artifact reduction algorithm, the CT value ranges and the noise were substantially reduced (1815.3 \pm 793.7 versus $231.7 \pm 95.9$ and $319.9 \pm 136.6$ versus $45.9 \pm 14.0$; both $P<.001$ ) regardless of the types of metallic objects and various sizes of coil masses. The rater study achieved an overall improvement of imaging quality and artifact reduction $(85.7 \%$ and $78.6 \%$ of cases by 2 raters, respectively), with the greatest improvement in the coiling group, moderate improvement in the liquid embolizers, and the smallest improvement in ventricular shunting (overall agreement, 0.857).

CONCLUSIONS: The metal artifact reduction algorithm substantially reduced artifacts and improved the objective image quality in every studied case. It also allowed improved diagnostic confidence in most cases.

ABBREVIATIONS: $C C F=$ carotid cavernous fistula; $F D C T=$ flat detector $C T ;$ MAR $=$ metal artifact reduction; $\mathrm{HU}=$ Hounsfield unit

$\mathbf{F}_{\mathrm{a}, \mathrm{s}}$ lat panel detectors have been used in radiography and fluoroscopy with the advantages of offering a higher dynamic range, dose reduction, direct digital readout, and a higher frame rate of dynamic image acquisition. ${ }^{1}$ Earlier reports about projection data by $\mathrm{C}$-arm-based flat detector-equipped angiography were pub-

Received September 17, 2013; accepted after revision November 18.

From the Department of Radiology (S.-C.H., C.-C.W., C.-J.L., W.-Y.G., C.-B.L., F.-C.C., C.-Y.C.), Taipei Veterans General Hospital, Taipei, Taiwan; and Department of Biomedical Imaging and Radiological Sciences (S.-C.H.) and School of Medicine (S.-C.H., C.-C.W., C.-J.L., W.-Y.G., C.-B.L., F.-C.C., C.-Y.C.), National Yang-Ming University, Taipei, Taiwan.

This work was supported, in part, by a collaborative project (T1100200) of Taipei Veterans General Hospital and Siemens. The MAR prototype was provided by Siemens.

Paper previously presented in part at: Annual Meeting of the Japanese Society of Neuroradiology, February 15-16, 2013; Kitakyushu, Japan.

Please address correspondence to WanYuo Guo, MD, PhD, No. 201, Sec 2, Shipai Rd, Beitou District, Taipei, Taiwan 11217; e-mail: wyguo@vghtpe.gov.tw

$\square$ Indicates article with supplemental on-line figures.

http://dx.doi.org/10.3174/ajnr.A3851 lished in the 2000s. ${ }^{2,3}$ With the advances of flat detector technology, the angiographic suites equipped with $\mathrm{C}$-arm flat detectors can generate $3 \mathrm{D}$ angiography and high-resolution volume images with soft-tissue contrast resolution. Today, the term "flat detector CT" (FDCT) refers to CT images with a series of projection data using a C-arm-based flat detector system, known as angiographic CT. ${ }^{4}$ This technology has gained popularity and provided a number of important clinical applications. ${ }^{5}$

As a crucial tool for follow-up examinations in the angiographic suite, FDCT detects most intraparenchymal hemorrhages in emergency situations ${ }^{6,7}$ and can recognize intracranial complications early during endovascular surgery, such as coiling of an intracranial aneurysm, stent placement, or other interventional procedures. ${ }^{7}$ However, severe artifacts stemming from metallic objects remarkably degrade the image quality of FDCT and prevent visualization of the adjacent brain parenchyma or hemorrhage. Prell et $\mathrm{al}^{8}$ described a metal artifact reduction (MAR) algorithm that significantly improved imaging quality in the FDCT of 7 patients. Further studies based on a previous MAR 

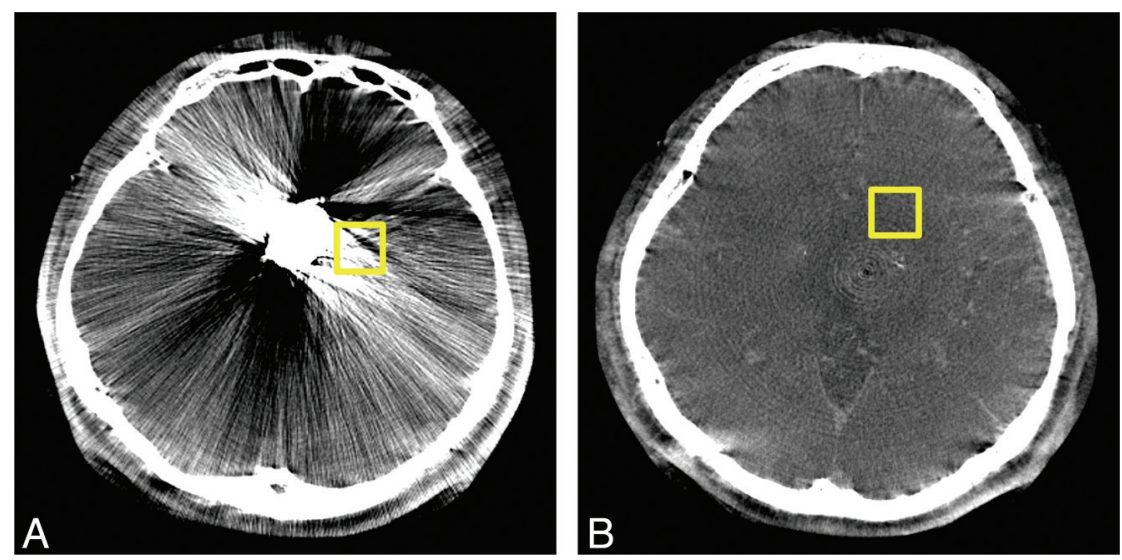

responsible for the artifacts. The data along the metal region boundaries are used to replace these data by a nonlinear interpolation procedure. The so-far corrected volume is used for a second normalized MAR correction step. ${ }^{11}$ This step includes, additionally, iterative improvements of the metal region boundaries to enhance the consistency of the corrected data as a whole. Finally, a procedure minimizing the total variation is applied to reduce residual streaks.

The uncorrected and corrected image volumes were reformatted to stacks of

FIG 1. Hounsfield unit value range and noise were measured by two $1.5 \times 1.5 \mathrm{~cm}^{2}$ square ROIs. The first region of interest (ROII) is placed in the region with most severe artifacts in the vicinity of the metal implant ( $A$ ). The second region of interest (ROIO) is placed in the section just above the target metal object, which is degraded by metallic artifacts $(B)$. transverse images with section thicknesses of $2.5 \mathrm{~mm}$ for the following analysis.

algorithm $^{8}$ have demonstrated the success in coiling and stentassisted coiling FDCT and CT angiography. ${ }^{9,10}$ However, the comparison of the efficacy of artifact reduction for various sizes and types of metal objects was seldom discussed. In this study, we investigated whether a MAR prototype software could reduce artifacts stemming from different high-attenuation implants and improve the diagnostic confidence of radiologists.

\section{MATERIALS AND METHODS}

Our institutional review board approved this study. We retrospectively reprocessed consecutive postinterventional FDCT images of 28 patients ( 15 men, 13 women; mean age, 55.6 years; range, 13-85 years) between January 2009 and November 2011. Twenty patients had aneurysms or carotid cavernous fistulas (CCFs) and were treated with endovascular coiling and stent placement. One patient had a left CCF, treated with 35\% n-BCA. Five patients had cerebral AVMs or dural arteriovenous fistulas and were treated with Onyx (Covidien, Irvine, California). Two had hydrocephalus and were treated with ventricular shunt drainage.

All images were obtained from a flat panel angiographic Carm system (Axiom Artis; Siemens, Erlangen, Germany) with the same FDCT protocol. The high-quality FDCT scan protocol included an acquisition range of $200^{\circ}$ (20 seconds for acquisition, $1240 \times 960$ projection matrix, $0.4^{\circ}$ angular increment) and 496 projections. The uncorrected FDCT images were reconstructed by using the dedicated software syngo InSpace 3D (Siemens).

All images were then postprocessed by using an MAR prototype software on an off-line workstation (MAR_VB21, Version 10; Siemens). The MAR algorithm investigated in this work is a modification and, additionally, an extension of a recently published MAR algorithm. ${ }^{8}$ The algorithm consists of several steps. Initially, an uncorrected volume image is reconstructed from the measured data. By segmenting the metal objects in this volume, one can obtain a binary metal volume image. For each projection, this binary volume is forward-projected to yield a binary projection image of metal regions on the detector in the respective position. The projection data contained in these metal regions are generated by rays through metal objects, and thus these data are

\section{Image Analysis and Statistical Analysis}

Objective Assessment. An experienced neuroradiologist (S.-C.H.) placed the first region of interest (ROI1) of $1.5 \times 1.5$ $\mathrm{cm}^{2}$ in exactly the same location of the brain parenchyma closest to the metal objects in both uncorrected and corrected images. A second same-size region of interest (ROI0) was placed in the parenchyma in the section above or below the metal artifacts, which was used for the brain parenchyma reference without metal artifact interference (Fig 1).

The range of Hounsfield unit (HU) values in the region of interest was defined as the difference of maximal ( $\max$ ) and minimal (min) values,

$$
\mathrm{HU} \text { range }=\mathrm{HU}_{\max }-\mathrm{HU}_{\text {min }} \text {, }
$$

and the noise was defined as the SD of HU values in the region of interest.

Subjective Assessment. Two neuroradiologists (S.-C.H. and C.-C.W.) evaluated each examination independently on the commercialized PACS viewer of our hospital. All images were anonymized; the raters were blinded to patient information and types of metal objects. The raters compared uncorrected and corrected images according to the artifacts around the metal objects and rated improved diagnostic confidence by using a 5-score system: $(-1=$ worse metal artifacts, $0=$ no substantial change of metal artifacts, $1=$ mild reduction of artifacts but not helpful for the diagnosis, 2 = marked reduction of metal artifacts and improved diagnostic confidence, $3=$ no residual artifacts).

\section{Statistical Analysis}

We divided the patients into 3 groups according to the metal objects, including coiling \pm stent placement, liquid embolizers ( $n$-BCA or Onyx), and shunts. The coiling group was further divided into 3 subgroups according to the coil lengths $(<25 \mathrm{~cm}, \geqq 25 \mathrm{~cm}$ but $<100$ $\mathrm{cm}, \geqq 100 \mathrm{~cm}$ ). We used paired $t$ tests to compare the range and noise of ROI1 between uncorrected and corrected images and between ROI0 and ROI1 in the corrected images. We calculated the overall interrater agreement by raw statistics and analyzed the reliability of the agreement by using $\kappa$ statistics. We used the $\chi^{2}$ and Fisher exact 
Table 1: Quantitative assessment of metal artifacts before and after reduction

\begin{tabular}{|c|c|c|c|c|c|c|c|c|}
\hline \multirow[b]{2}{*}{ Objects } & \multicolumn{3}{|c|}{ HU Range } & \multirow[b]{2}{*}{$P$ Value } & \multicolumn{3}{|c|}{ Noise } & \multirow[b]{2}{*}{$P$ Value } \\
\hline & Before & After & $\%$ Change & & Before & After & $\%$ Change & \\
\hline ROIO ( $n=28)$ & $247.2 \pm 37.5$ & $247.3 \pm 37.3$ & 0.04 & NS & $36.3 \pm 4.8$ & $36.2 \pm 4.7$ & 0.2 & NS \\
\hline Coiling \pm stenting $(n=20)$ & $2005.6 \pm 804.0$ & $282.6 \pm 55.8$ & 85.9 & $<.001$ & $251.7 \pm 94.4$ & $42.9 \pm 10.3$ & 82.9 & $<.001$ \\
\hline$<25 \mathrm{~cm}(n=6)$ & $1613.7 \pm 709.7$ & $296 \pm 44.7$ & 81.7 & .006 & $198.4 \pm 83.8$ & $42.8 \pm 7.3$ & 78.4 & .007 \\
\hline $25-100 \mathrm{~cm}(n=7)$ & $1954.9 \pm 634.2$ & $257.6 \pm 23.5$ & 86.8 & $<.001$ & $238.6 \pm 49.3$ & $38.9 \pm 5.6$ & 83.7 & $<.001$ \\
\hline$>100 \mathrm{~cm}(n=7)$ & $2392.3 \pm 945.6$ & $296 \pm 80.7$ & 87.6 & .001 & $310.3 \pm 113.5$ & $46.9 \pm 15.0$ & 84.9 & .001 \\
\hline Liquid embolizer $(n=6)$ & $1377.3 \pm 646.6$ & $428.5 \pm 259.0$ & 68.9 & .019 & $206.0 \pm 86.3$ & $56.9 \pm 21.6$ & 72.3 & .007 \\
\hline Shunt ( $n=2)$ & $1226.5 \pm 259.5$ & $368.0 \pm 76.4$ & 70.9 & NS & $109.8 \pm 2.6$ & $44.0 \pm 0.7$ & 60 & .013 \\
\hline Overall (ROII) $(n=28)$ & $1815.3 \pm 793.7$ & $319.9 \pm 136.6$ & 82.3 & $<.001$ & $231.7 \pm 95.9$ & $45.9 \pm 14.0$ & 80.2 & $<.001$ \\
\hline
\end{tabular}

Note:-NS indicates no significance.
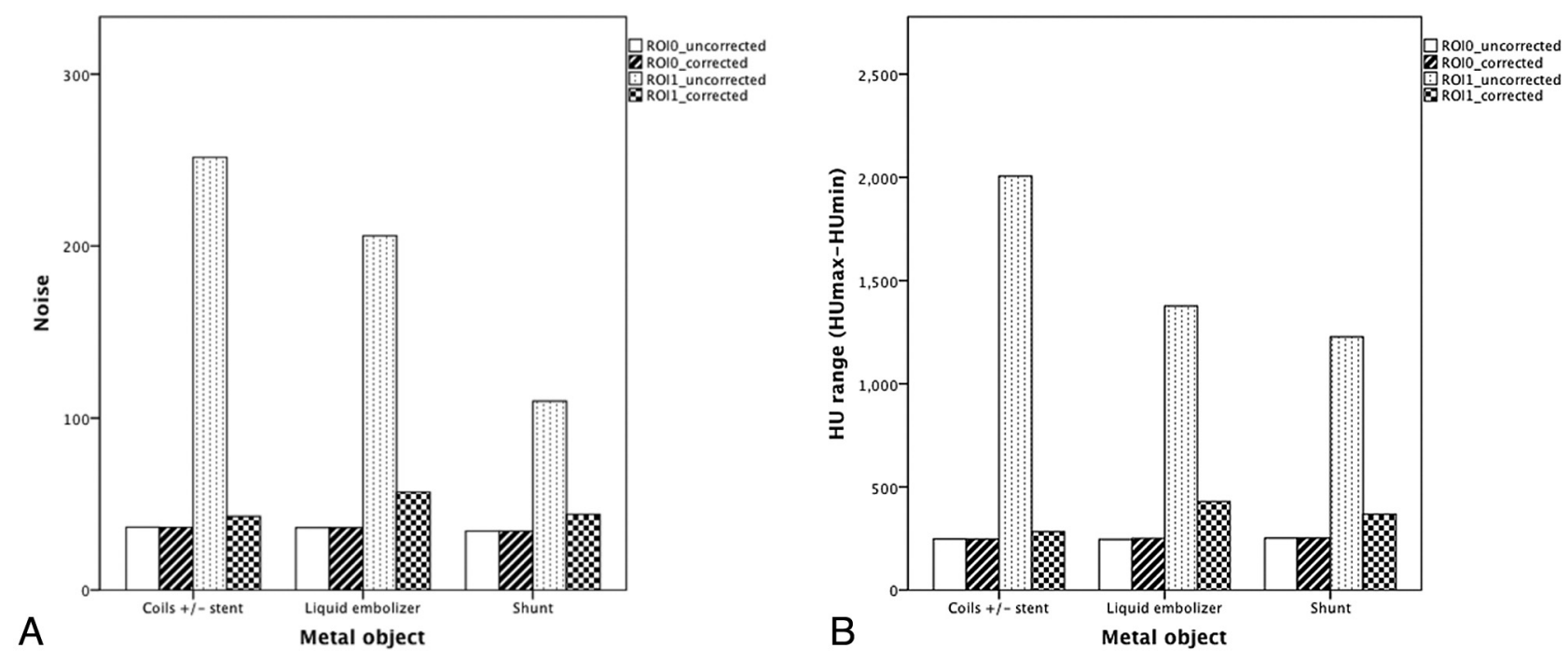

FIG 2. A, Comparison of Hounsfield unit range among different objects. B, Comparison of noise among different metal objects.
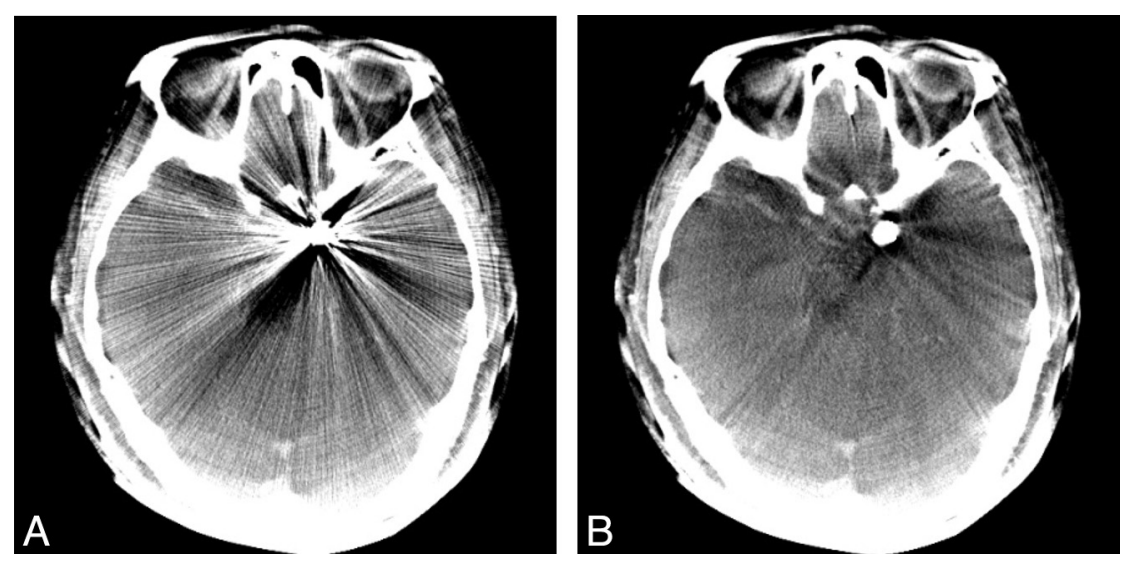

FIG 3. Illustrative case 1. FDCT without MAR $(A)$ and with MAR $(B)$ of a left-side ICA aneurysm post-coil embolization. The metal artifacts are significantly reduced, and the postcorrected images enable clearer evaluation of adjacent parenchyma.

tests to calculate the significance of the results. A 2-tailed level of $P<.05$ was considered significant.

\section{RESULTS}

The images of all patients demonstrated streak artifacts and were processed by the MAR algorithm successfully. The results are summarized in Table 1.

\section{Comparison among Metal Objects}

The application of the MAR algorithm did not compromise the image quality in the section without metal artifact contamination

\section{Comparison among Various Coil Lengths}

In the coiling group, the Hounsfield unit range and noise of ROI1 were related to the coil length before the MAR algorithm correction; however, no significant difference existed (Fig 4). A significant reduction of metal artifacts was achieved in all patients with different metal objects $(P<.05)$. Except for the noise in the subgroup length between 25 and $100 \mathrm{~cm}$, the Hounsfield unit range and noise of ROI1 in the corrected images showed no significant difference compared with those of ROI0 in the other subgroups (Fig 3). 

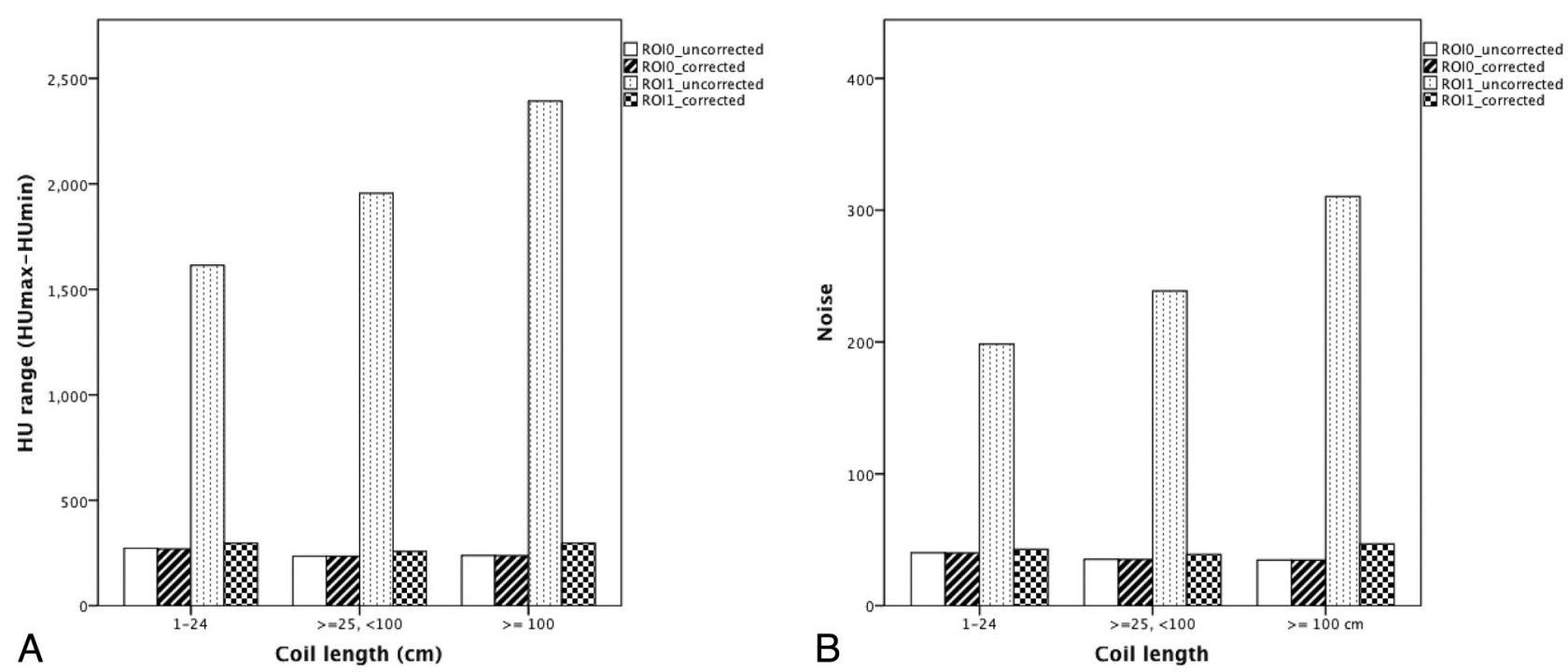

FIG 4. A, Comparison of Hounsfield unit range among different coil lengths. $B$, Comparison of noise among different coil lengths.

Table 2: Results of the observer study rating the imaging quality with MAR

\begin{tabular}{|c|c|c|c|c|c|c|c|c|c|c|c|c|}
\hline \multirow[b]{2}{*}{ Objects } & \multicolumn{5}{|c|}{ Rater 1 Score } & \multirow[b]{2}{*}{$P$ Value } & \multicolumn{5}{|c|}{ Rater 2 Score } & \multirow[b]{2}{*}{$P$ Value } \\
\hline & -1 & 0 & 1 & 2 & 3 & & -1 & 0 & 1 & 2 & 3 & \\
\hline Coiling \pm stenting & 0 & 0 & 0 & 18 & 2 & & 0 & 0 & 1 & 13 & 6 & \\
\hline Liquid embolizer & 0 & 0 & 2 & 4 & 0 & & 0 & 0 & 3 & 3 & 0 & \\
\hline Shunt & 0 & 0 & 2 & 0 & 0 & & 0 & 2 & 0 & 0 & 0 & \\
\hline Overall & 0 & 0 & 4 & 22 & 2 & .002 & 0 & 2 & 4 & 16 & 6 & $<.001$ \\
\hline
\end{tabular}

Table 3: Inter-rater agreement of the observer study

\begin{tabular}{lcccc}
\hline & $\begin{array}{c}\text { Overall } \\
\text { Agreement }\end{array}$ & $\begin{array}{c}\text { Rater 1, } \\
\text { Score } \leq \text { 2(\%) }\end{array}$ & $\begin{array}{c}\text { Rater 2, } \\
\text { Score } \leq 2(\%)\end{array}$ & $\boldsymbol{\kappa}$ \\
\hline $\begin{array}{l}\text { Overall imaging } \\
\text { quality }\end{array}$ & 0.857 & 85.7 & 78.6 & .517 \\
\hline
\end{tabular}

\section{Subjective Assessment of Image-Quality Improvement}

The results of the observer study are summarized in Tables 2 and 3. The overall agreement of the 2 raters was $85.7 \%(\kappa=0.517)$. No case had more severe artifacts or worse imaging quality after MAR correction. The number of cases in which the raters agreed on significant artifact reduction and improved image quality (score of $\geq 2$ ) was $>95 \%$ in the coiling group, $50 \%$ in the liquid embolizer group, and none in the shunt group.

\section{DISCUSSION}

An angiographic suite equipped with flat panel detectors can provide in-room CT-like images before or after neurointerventional procedures without transferring patients to another conventional CT scanner. These images can identify the relationship of the metal implants and provide early recognition of rebleeding or intracranial procedure-related complications, such as incomplete stent deployment, stent migration, stent fracture, or coil dislocation. ${ }^{12-14}$ However, the metallic artifacts stemming from metal objects hinder clear visualization of a hematoma or parenchyma surrounding metal objects. ${ }^{14}$

Prell et $\mathrm{al}^{15}$ demonstrated successfully reducing metal artifacts by using their version of a MAR algorithm in a small series of 7 patients who had cerebral aneurysms and were treated with coiling or clipping. Other studies, based on their own versions of a
MAR algorithm, have further demonstrated the visualization usefulness of the stent strut and parent vessels in stent-assisted coiling. ${ }^{9,10}$ However, the efficacy of the MAR algorithm, as described above in managing different metal objects, has not been reported, to our knowledge.

The patients in our study did not undergo FDCT before the operation. Therefore, we chose the brain parenchyma in a higher or lower section, which was free of metallic artifacts, as the internal reference of imaging quality. We demonstrated that the Siemens MAR software remarkably reduces artifacts and corrects image quality to a level close to the imaging quality of the native image by using adjacent parenchyma as the internal reference. Although the Hounsfield unit range and noise of the corrected images remained slightly higher than those of native images, no significant differences existed. We noticed that in most cases, certain darker areas surrounding the implants remained after MAR correction. These artifacts may be the secondary artifacts introduced by the correction scheme and may be the reason the imaging quality remained inferior to that of native images. ${ }^{15,16}$ In our results, the types of metal objects and the coil lengths did not affect the results of the investigated MAR algorithm.

In assessing subjective image quality, we demonstrated that the MAR software substantially improved the visibility of the metal object vicinity, in which image qualities were most severely degraded before correction. Consequently, the overall imaging quality of surrounding regions improved in $>78 \%$ of the cases following MAR. Nearly all coiling cases had increased diagnostic confidence following MAR. In contrast, the rater score was lower in the liquid embolizer group, though the Hounsfield unit range and noise in the corrected images showed no difference from 
those of the coiling group. Our explanation is that liquid embolizer shape and distribution were typically irregular and scattered in these cases. Despite significant artifacts reduction, the blurred regions and the secondary artifacts introduced by the replaced imaging content of the original $\mathrm{x}$-ray data accumulated in the central regions and significantly hindered the image interpretation (Online Fig 1). Therefore, only half of the cases achieved higher diagnostic confidence following MAR. None of the ventricular shunting group provided additional diagnostic information following MAR because the shunt artifacts were typically limited to the tip of the shunt and were minor, which explains why most cases could be interpreted easily without requiring MAR correction (Online Fig 2).

The main limitation of our study was the relatively small number of cases. We only analyzed 6 patients receiving liquid embolizers and 2 patients undergoing ventricular drainage. Although the sample size was small, the MAR application resulted in promising results for correcting metal artifacts and improving image quality, indicating the need for further research. In the coiling group, we chose coil length rather than aneurysm packing attenuation as the coil attenuation parameter. Because the coil masses are irregular and bilateral in CCF cases, it is difficult to apply the formula of the aneurysm packing attenuation in these cases. Our results indicate the correlation of coil length with artifact severity and achieving reduced metal artifacts in all subgroups of different coil lengths. These findings may provide indirect evidence that the MAR software successfully reduces metal artifacts, regardless of various coiling densities.

\section{CONCLUSIONS}

We demonstrated using the MAR prototype to reduce streak artifacts substantially around different metallic implants on FDCT and to improve objective and subjective imaging quality.

Disclosures: Sheng-Che Hung-RELATED: Provision of Writing Assistance, Medicines, Equipment, or Administrative Support: MAR prototype software, ${ }^{\star}$ Comments: collaborative project (T1100200) of the Taipei Veterans General Hospital and Siemens, the MAR. Wan-You Guo-RELATED: Other: supported in part by a collaboration research contract between Taipei Veterans General Hospital and Siemens. *Money paid to the institution.

\section{REFERENCES}

1. Kalender WA, Kyriakou Y. Flat-detector computed tomography (FD-CT). Eur Radiol 2007;17:2767-79

2. Akpek S, Brunner T, Benndorf G, et al. Three-dimensional imaging and cone beam volume $\mathrm{CT}$ in $\mathrm{C}$-arm angiography with flat panel detector. Diagn Interv Radiol 2005;11:10-13

3. Kalender WA. The use of flat-panel detectors for CT imaging [in German]. Radiologe 2003;43:379-87

4. Gupta R, Cheung AC, Bartling SH, et al. Flat-panel volume CT: fundamental principles, technology, and applications. Radiographics 2008;28:2009-22

5. Hung SC, Lin CJ, Guo WY, et al. Toward the era of a one-stop imaging service using an angiography suite for neurovascular disorders. Biomed Res Int 2013;2013:873614

6. Struffert T, Richter G, Engelhorn T, et al. Visualisation of intracerebral haemorrhage with flat-detector CT compared to multislice CT: results in 44 cases. Eur Radiol 2009; 19:619-25

7. Dörfler A, Struffert T, Engelhorn T, et al. Rotational flat-panel computed tomography in diagnostic and interventional neuroradiology. Rofo 2008;180:891-98

8. Prell D, Kalender WA, Kyriakou Y. Development, implementation and evaluation of a dedicated metal artefact reduction method for interventional flat-detector CT. Br J Radiol 2010;83:1052-62

9. van der Bom IM, Hou SY, Puri AS, et al. Reduction of coil mass artifacts in high-resolution flat detector conebeam CT of cerebral stent-assisted coiling. AJNR Am J Neuroradiol 2013;34:2163-70

10. Psychogios MN, Scholz B, Rohkohl C, et al. Impact of a new metal artefact reduction algorithm in the noninvasive follow-up of intracranial clips, coils, and stents with flat-panel angiographic CTA: initial results. Neuroradiology 2013;55:813-18

11. Meyer E, Raupach R, Lell M, et al. Normalized metal artifact reduction (NMAR) in computed tomography. Med Phys 2010;37:5482-93

12. Mordasini P, Al-Senani F, Gralla J, et al. The use of flat panel angioCT (DynaCT) for navigation through a deformed and fractured carotid stent. Neuroradiology 2010;52:629-32

13. Buhk JH, Kallenberg K, Mohr A, et al. Evaluation of angiographic computed tomography in the follow-up after endovascular treatment of cerebral aneurysms-a comparative study with DSA and TOF-MRA. Eur Radiol 2009;19:430-36

14. Clarençon F, Piotin M, Pistocchi S, et al. Evaluation of stent visibility by flat panel detector CT in patients treated for intracranial aneurysms. Neuroradiology 2012;54:1121-25

15. Prell D, Kyriakou Y, Struffert T, et al. Metal artifact reduction for clipping and coiling in interventional C-arm CT. AJNR Am J Neuroradiol 2010;31:634-39

16. Prell D, Kyriakou Y, Beister M, et al. A novel forward projectionbased metal artifact reduction method for flat-detector computed tomography. Phys Med Biol 2009;54:6575-91 